\title{
A conceptual approach for noncontact calorimetry in space
}

\author{
H. J. Fecht and W. L. Johnson \\ Universität Augsburg, Institut für Physik, D-8900 Augsburg, FRG and \\ W. M. Keck Laboratory of Engineering Materials, California Institute of Technology, Pasadena, \\ California 91125
}

(Received 30 April 1990; accepted for publication 17 September 1990)

\begin{abstract}
A concept is developed and described which allows to measure the heat capacity and the effective thermal conductivity of stable and undercooled liquid metals and alloys in an electromagnetic levitation apparatus. We propose to use an ac pulse heating method which is used nowadays as a standard technique for precision measurement of low temperature heat capacities. The ideal process parameters including the drop diameter $D$, temperature $T$, and frequency of measurement $\omega$ can be optimized when the following relations hold for the external and internal relaxation time constants $\tau_{1}$ and $\tau_{2}$, respectively: $\omega \tau_{1}>10$ and $\omega \tau_{2}<0.1$. Then heat capacity data can be obtained with an accuracy of better than $1 \%$ with $D$ about 5 to $10 \mathrm{~mm}, T$ between 1200 and $1800 \mathrm{~K}$ and $\omega$ between 0.1 and $1 \mathrm{~Hz}$ for typical metals and alloys.
\end{abstract}

\section{INTRODUCTION}

Calorimetric data, in particular the specific heat and the thermal conductivity coefficients, are crucial in evaluating the nucleation and growth kinetics during solidification from an undercooled liquid. ${ }^{1}$ The temperature dependence of the difference in specific heat between the crystalline and undercooled liquid phases $\left(\Delta c_{p}\right)$ can be measured with samples prepared by emulsification techniques. Especially for glass forming alloys, large deviations of the specific heat of the undercooled liquid from an ideal behavior $\left(\Delta c_{p}=0\right)$ have been observed at intermediate temperatures $(<650 \mathrm{~K}))^{2,3}$ This has a critical impact on the analysis of nucleation kinetics during solidification where an exact model for the driving force (Gibbs free energy difference) is needed. The use of different models for the driving force based on different approaches for the heat capacity $\left[\Delta c_{p}=0, \Delta c_{p}=\right.$ const., $\left.\Delta c_{p}=\Delta c_{p}(T)\right]$, consequently leads to large variations in the prefactors ( $\mathrm{six}$ orders of magnitude) of the classical equation for the nucleation frequency. ${ }^{3}$ Therefore, the analysis of nucleation kinetics without accurate knowledge of the energies is circular in its argument and can give misleading results.

For metals and alloys with high melting points, specific heat data for both the equilibrium and the undercooled liquid are virtually unavailable. Similarly, thermal conductivity data on liquids of high melting point metals are scarce and difficult to obtain. Therefore, we propose a method which allows the accurate determination of the heat capacity as well as the thermal conductivity of metastable undercooled liquid metals and alloys using a magnetic levitation system, i.e., TEMPUS (tiegelfreies elektromagnetisches prozessieren unter schwerelosigkeit).

\section{AC METHOD FOR SPECIFIC HEAT AND THERMAL CONDUCTIVITY MEASUREMENT}

Measurements of the specific heat $C$ are traditionally performed by adiabatic calorimetry methods. Such calorimeters are isolated from the environment and require long relaxation times. When heat is supplied to the sample, the heat capacity is determined from the resulting temperature rise. Such methods are difficult or impossible to use for noncontact measurements at high temperature. The reactivity of high-temperature melts further makes containerless processing an attractive option.

With the development of lock-in amplifiers, a new kind of calorimetry became attractive, the ac temperature method. ${ }^{4,5}$ This method was originally developed in conjunction with low-temperature heat capacity measurements. However, the principles can be applied more generally. With this method, the required relaxation times are much shorter and this method becomes feasible for noncontact calorimetry. The basic ideas and concepts are described in the following.

In its original conception, the ac method involves a sample connected to a thermal reservoir at temperature $T_{o}$, to a thermocouple and a heater through thermal conductances $K_{b}, K_{\theta}$, and $K_{h}$, respectively. The sample is otherwise isolated as shown in Fig. 1 . Now an ac signal $\boldsymbol{P}_{\omega}$ is applied to the heater in intimate thermal contact with the sample. This produces a power signal of the form:

$$
P_{\omega}=P_{\omega 0} \cos ^{2}(\omega t / 2) \text {. }
$$

If the internal thermal conductivity of the sample is finite, then there will generally be temperature gradients within the sample induced by this ac signal. The time scale for relaxation of such gradients is given by:

$$
\tau_{2}=C / K_{\text {int }},
$$

where $K_{\text {int }}$ is an internal thermal conductance for the sample and $C$ is the total heat capacity of the sample (in $\mathrm{J} / \mathrm{K}$ ). In general, the ac signal will produce a periodic variation of the sample temperature. The form of the variation can be determined by solving the appropriate differential equations. ${ }^{4-6}$

If the sample were initially at temperature $T_{o}+\Delta T$ in the absence of an ac signal, it would relax (for small $\Delta T$ ) to temperature $T_{o}$ exponentially according to the equation: 


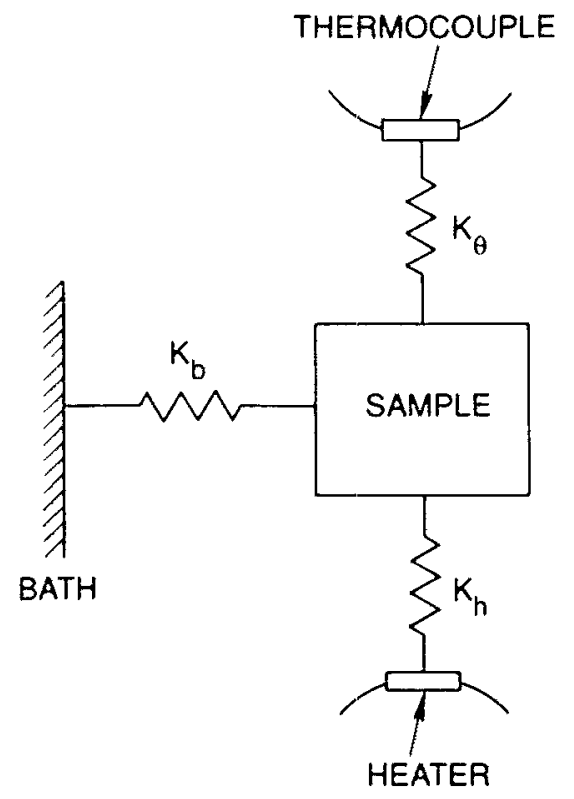

FIG. 1. Schematic diagram of ac heat capacity measurement with the sample coupled to a bath, thermometer, and heater by the thermal conductances $\mathbf{K}_{b}, \mathbf{K}_{b}$, and $\mathbf{K}_{h}$, respectively.

$$
\Delta T(t)=\exp \left(-t / \tau_{1}\right)
$$

where

$$
\tau_{1}=C / K_{b}
$$

In the presence of the ac signal, it can be shown $n^{4,5}$ that the amplitude of the ac temperature modulation at the heater location or the sample is given by:

$$
\begin{aligned}
(\Delta T)_{\omega}= & P_{\omega 0} / 2 \omega C\left[1+\left(\omega \tau_{1}\right)^{-2}+\left(\omega \tau_{2}\right)^{2}\right. \\
& \left.+2 K_{b} / 3 K_{s}\right]^{-1 / 2} .
\end{aligned}
$$

If $\omega$ is properly chosen, the effect of the second and third terms in the bracket can be minimized. For instance, if

$$
\omega \tau_{1} / 10>1>10 \omega \tau_{2},
$$

then, with a relative accuracy of $1 \%$, the ac temperature signal will be given by

$$
(\Delta T)_{\omega}=P_{\omega 0} / 2 \omega C \text {. }
$$

If $P_{\omega 0}$ is known, and $(\Delta T)_{\omega}$ is measured, then

$$
C=P_{\omega 0} / 2 \omega(\Delta T)_{\omega},
$$

and the sample heat capacity can be determined.

\section{NONCONTACT CALORIMETRY}

We now turn to the question of applying this technique to the problem of noncontact calorimetry. We assume a metal droplet of spherical shape with radius $R$ which is levitated or positioned in an apparatus (such as the TEMPUS facility) and maintained at a constant temperature $T_{o}$ by a fixed power input $P_{o}$ supplied by a rf field of a

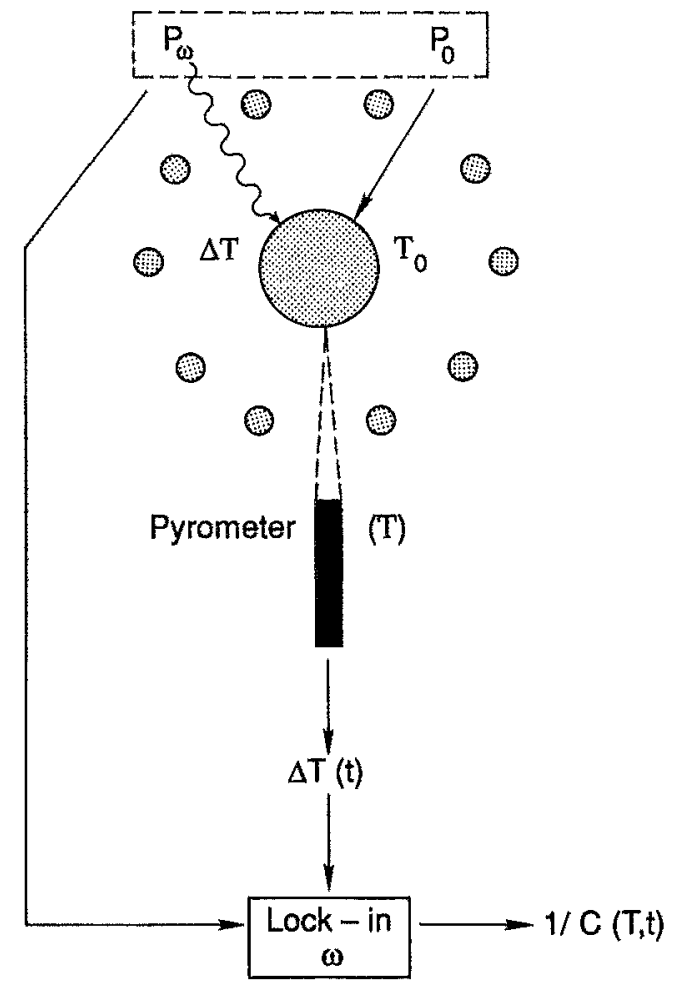

FIG. 2. Schematic diagram of noncontact calorimetry where the liquid drop is levitated within an electro-magnetic coil.

heating coil. The situation is depicted in Fig. 2. Under ideal conditions, the sample is located at the center of a symmetrically shaped heating coil and does not move or change shape during the course of the experiment. We begin with this ideal situation (We will later return to the problem of sample motion and take account of its effect on measurement.) Under such ideal conditions, the power input, $P_{o}$, to the sample can be assumed to be time independent and the sample temperature in steady state is determined by balancing the heat loss from the sample against the power input. There exist two principal mechanisms for heat loss. These are

(i) Radiation

$$
P_{\mathrm{rad}}=A \epsilon \sigma\left(T^{4}-T_{\mathrm{en}}^{4}\right),
$$

where

$A=$ Sample surface area,

$\sigma=$ Stefan-Boltzmann constant $\left[0.57 \times 10^{-11}\right.$ Watts $/$ $\left.\left(\mathrm{cm}^{2}-\mathrm{K}^{4}\right)\right]$,

$\epsilon=$ Total hemispherical emissivity of the sample surface,

$T_{\mathrm{en}}=$ Temperature of the environment (apparatus chamber).

Under the conditions of our experiment, it will turn out that we can ignore the energy transfer from the chamber at ambient temperature to the sample through the radiation field (second term above).

(ii) Heat conduction

$$
P_{\text {cond }}=K_{m}\left(T-T_{\text {en }}\right),
$$


where

$$
\begin{gathered}
K_{m}=\text { Thermal conductance of the medium (gas) } \\
\text { in the chamber }
\end{gathered}
$$

We may neglect this heat loss mechanism if the vacuum in the chamber is better than $10^{-5}$ Torr. For the TEMPUS facility, the pressure will be $10^{-9}$ Torr. This heat loss mechanism will thus not be considered in what follows.

Under the above assumptions, the droplet will be in steady state equilibrium at temperature $T_{o}$ when

$$
P_{o}=A \epsilon \sigma T_{\circ}^{4}
$$

or when

$$
T_{o}=\left[P_{o} /(A \epsilon \sigma)\right]^{1 / 4} \text {. }
$$

If $T_{o}, A$, and $P_{o}$ can be measured, then the total emissivity $\epsilon$ of the sample can be determined. In practice, for the TEMPUS facility, we could determine $P_{o}$ (the rf power coupled to the sample) as a function of the rf voltage, $V_{\mathrm{rf}}$, applied to the heating coils of TEMPUS, and the corresponding temperature $T_{0}$ using a black-body bolometer $(\epsilon=1)$.

One might immediately ask whether or not temperature gradients will exist in the droplet under these steadystate conditions. Such temperature gradients might arise if the rf power is coupled to the spherical droplet in a nonsymmetrical manner. This will depend on an internal relaxation time constant of the sample (and thus on the size and thermal conductivity of the sample). We can calculate an internal relaxation time for the sample if we have the effective thermal conductance $K_{\text {int }}$ for the particular mode of the temperature gradient distribution. We will not consider the full problem here but rather make an estimate of $K_{\text {int }}$. The only relevant dimension of the droplet is its radius. The average area through which heat is transported radially is

$$
\langle A\rangle=R^{-1} \int_{0}^{R} 4 \pi R^{2} d R=(4 \pi / 3) R^{2},
$$

while the characteristic distance of transport is $R$. If the thermal conductivity of the droplet is $\kappa$, then we have for the first harmonics of the heat flow

$$
K_{\mathrm{int}}=\gamma(\langle A\rangle / R) \kappa=4 \pi R \gamma \kappa / 3,
$$

where $\gamma$ is a geometrical factor which equals $\pi^{2}$ for a spherical sample. ${ }^{6}$ This gives an internal relaxation time for temperature gradients of the first harmonics of

$$
\tau_{2}=\text { internal relaxation time }=C / K_{\text {int }}=3 C / 4 \pi R \gamma \kappa .
$$

We must now determine the effective external relaxation time for the droplet. This is the characteristic decay time for the temperature of the droplet to its steady state value when its initial value is perturbed from the steady state. In the absence of an ac signal, the time dependence of the temperature of the droplet is given by

$$
d T / d t=\left(P_{o}-A \epsilon \sigma T_{o}^{4}\right) / C .
$$

For steady state, we have

$$
d T / d t=0
$$

and

$$
P_{o}=A \epsilon \sigma T_{o}^{4}
$$

If the initial temperature differs from $T_{o}, T_{i}=T_{0}$ $+\Delta T$, and $\Delta T$ is small compared to $T_{o}$, then we will have

$$
\begin{aligned}
d(\Delta T) / d t= & \left.d\left(P_{o}-A \epsilon \sigma T_{o}^{4}\right) d t\right|_{T=T_{o} \Delta T} \Delta t \\
& +\ldots(\text { higher order terms })
\end{aligned}
$$

or

$$
d(\Delta T) / d t=-\left(4 A \epsilon \sigma T_{o}^{3}\right) \Delta T / C
$$

which has the solution

$$
\begin{aligned}
& \Delta T(t)=\exp (-\lambda t)=\exp \left(-t / \tau_{1}\right) \\
& \text { with } \lambda=4 A \epsilon \sigma T_{o}^{3} / C .
\end{aligned}
$$

The external relaxation time $\tau_{1}$ is thus

$$
\tau_{1}=C /\left(4 A \epsilon \sigma T_{o}^{3}\right) \text {. }
$$

We propose to carry out ac calorimetry on our droplet. The principle is the same as that described earlier. In addition to the constant power $P_{o}$ required to maintain steady state equilibrium, we will apply a time varying power to the droplet. This could be accomplished by suitable modulation of the heating power (in the TEMPUS facility). It could also be accomplished by using a supplemental heating source such as a $\mathrm{CO}_{2}$ laser (required power output of 5-10 W). For mathematical convenience, we assume that the form of the ac signal is

$$
P_{\omega}(t)=P_{\omega 0} \cos ^{2}(\omega t / 2) \text {. }
$$

This form allows us to directly carry over the results of Refs. 4 and 5. Thus, the total power input to the droplet is given by

$$
P=P_{0}+P_{\omega}(t) \text {. }
$$

In general, the total ac temperature variation induced in the drop by the ac signal in the absence of a coupling between the sample and the heater, bath and thermocouple (noncontact calorimetry) is given by

$$
(\Delta T)_{\omega}=P_{\omega 0} / 2 \omega C\left[1+\left(\omega \tau_{1}\right)^{-2}+\left(\omega \tau_{2}\right)^{2}\right]^{-1 / 2} \text {. }
$$

When the condition

$$
\omega \tau_{1} / 10>1>10 \omega \tau_{2}
$$

is satisfied, we will have (to an accuracy of $1 \%$ )

$$
C=2 \omega(\Delta T)_{\omega} / P_{\omega 0} \text {. }
$$

Thus, by the proper choice of the frequency $\omega$ of the ac power, it is possible to minimize the correction terms in Eq. (23) which allows accurate measurement of the heat capacity. When the condition of Eq. (6) is not satisfied, then $2 \omega(\Delta T)_{\omega} / P_{\omega 0}$ will become frequency dependent. This frequency dependence can be exploited in fact to determine the effective thermal conductivity in a microgravity environment and is discussed below. 


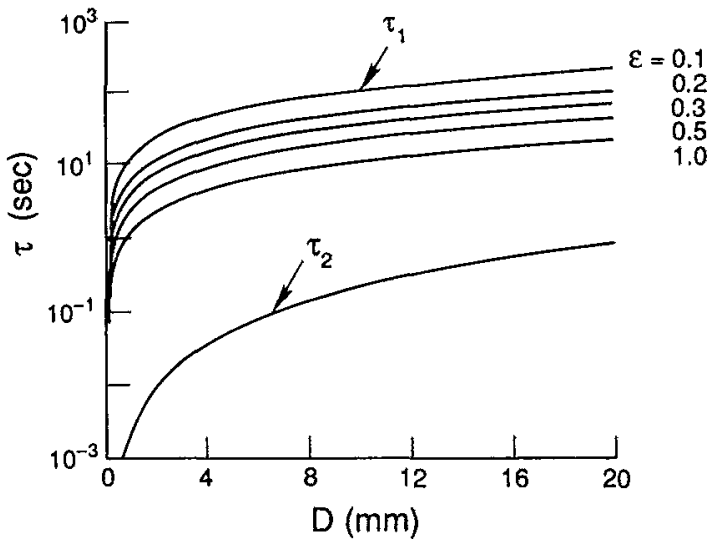

FIG. 3. The external relaxation time $\tau_{1}$ and internal relaxation time $\tau_{2}$ (on a logarithmic scale) for a liquid metal drop as function of diameter with $T_{o}=1500 \mathrm{~K}$ and the emissivity varied between $\epsilon=1$ and $\epsilon=0.1$.

\section{EXAMPLE AND REQUIREMENTS}

A similar method has been recently successfully applied for the heat capacity measurement of submillimeter size single carbon particles using a $\mathrm{CO}_{2}$ laser as heating source in an electrodynamic balance. ${ }^{7}$ Because of the small sample size an accuracy of $10 \%$ has been achieved with this method. By fine tuning the frequency and chosing larger samples (metals) in an electromagnetic balance, the accuracy of $c_{p}$ determination can be improved significantly. For the heat capacity measurement, the frequency $\omega$ of the modulated power is chosen, so that the two correction terms in Eq. (23) become minimal. Then the measured temperature variation $(\Delta T)_{\omega}$ and the power amplitude $P_{\omega 0}$ give the heat capacity $C$ [Eq. (8)] as described below.

The input power $P_{o}$ can be adjusted and the drop is stabilized in the rf coil by additional means (DC magnetic damping field). Under microgravity conditions (acceleration $=\mu g=$ independent of time), this guarantees a minimum disturbance of the drop. $P_{\omega 0}$ can be calibrated and determined in the UHV chamber by the radiative power transfer [Eq. (9)] to a black body located near the sample (black body bolometer). The temperature $T$ and the temperature variation $(\Delta T)_{\omega}$ are measured with a high precision pyrometer. An output signal of the pyrometer could be fed to a lock-in amplifier using a signal from the ac modulation of the rf power $\left[P_{\omega}(t)\right]$ as a reference signal to the lock-in amplifier. The dc output of the lock-in amplifier would then give $(\Delta T)_{\omega}$, thus eliminating the external noise.

As an example, the optimum conditions for a successful experiment have been evaluated. For this estimate, typical values for the thermal conductivity and heat capacity of a liquid metal drop of interest (i.e., $\mathrm{Zr}$-alloys) are taken as $\kappa=0.2 \mathrm{~W} / \mathrm{cm}-\mathrm{K}^{8}$ and $C$ corresponding to the DulongPetit value of $3 k_{b}$ per atom. Figure 3 shows the calculated internal and external relaxation times $\tau_{2}$ and $\tau_{1}$ (on a $\log$ arithmic scale) for a liquid metal drop at $1500 \mathrm{~K}$ as function of its diameter according to Eqs. (15) and (20), re-

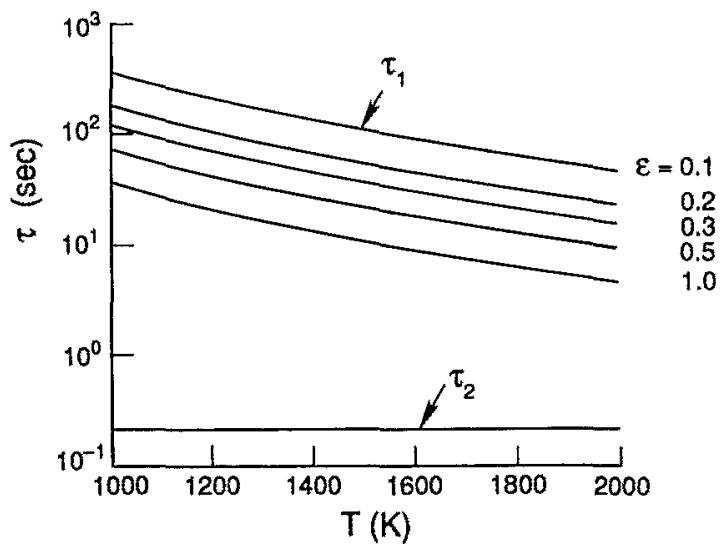

FIG. 4. The external relaxation time $\tau_{1}$ and internal relaxation time $\tau_{2}$ (on a logarithmic scale) for a liquid metal drop as function of temperature with $D=10 \mathrm{~mm}$ and the emissivity varied between $\epsilon=1$ and $\epsilon=0.1$.

spectively. The results suggest that the internal temperature gradients inside the liquid metal drop are relaxed within less than $1 \mathrm{~s}$. For emissivities varied between $\epsilon=1$ (black body) and $\epsilon=0.1$ (well polished surface) this results in a difference of up to three orders of magnitude for $\tau_{1}$ and $\tau_{2}$. [For many metals a typical emissivity $\epsilon$ of about 0.3 (independent of temperature) is found experimentally. $\left.{ }^{9}\right]$ The temperature dependence of these relaxation times is strong for $\tau_{1}$ (like $T^{-3}$ ) and very weak for $\tau_{2}$ [only $T$-dependence of $C(T)$ ]. This is depicted in Fig. 4 for a drop with $1 \mathrm{~cm}$ diam between 1000 and $2000 \mathrm{~K}$. The internal relaxation times as estimated here, are sufficiently short for accurate heat capacity measurements of liquid metals in contrast to nonconductive materials (ceramics and semiconductors).

The relaxation times $\tau_{1}$ and $\tau_{2}$ are the most critical parameters to achieve high accuracy in the heat capacity measurement. The frequency of the power modulation is chosen, so that the correction terms in Eq. (23) become negligible. This is shown in the following by estimating a correction factor

$$
\Delta=1-\left[1+\left(\omega \tau_{1}\right)^{-2}+\left(\omega \tau_{2}\right)^{2}\right]^{-1 / 2},
$$

which is a function of the heat capacity $C$ (which we want to measure), drop diameter $D$, temperature $T_{o}+(\Delta T)_{\omega}$, emissivity $\epsilon$ and thermal conductivity $\kappa$.

Figure 5 shows the correction factor $\Delta$ as function of frequency for (a) various diameters $(D=5,10,15 \mathrm{~mm})$ at $T_{o}=1500 \mathrm{~K}, \epsilon=0.3$ and $\kappa=0.2 \mathrm{~W} / \mathrm{cm}-\mathrm{K}$ (Fig. 5(a)], (b) various temperatures $\left(T_{o}=1200,1400,1600 \mathrm{~K}\right)$ at $D=10 \mathrm{~mm}, \epsilon=0.3$, and $\kappa=0.2 \mathrm{~W} / \mathrm{cm}-\mathrm{K}$ [Fig. $5(\mathrm{~b})$ ], (c) various emissivities $(\epsilon=0.2,0.3,0.5)$ at $T_{o}=1500 \mathrm{~K}$, $D=10 \mathrm{~mm}$, and $\kappa=0.2 \mathrm{~W} / \mathrm{cm}-\mathrm{K}$ [Fig. 5(c)], and (d) various thermal conductivities $(\kappa=0.1,0.2,0.4 \mathrm{~W} / \mathrm{cm}-\mathrm{K})$ at $D=10 \mathrm{~mm}, T_{o}=1500 \mathrm{~K}$, and $\epsilon=0.3$. From these figures it is seen, that higher accuracy of the $C$-measurement is obtained for smaller drop diameters, lower temperature, lower emissivity, and higher thermal conductivity. For frequencies in the range about $0.5 \mathrm{~Hz}$ the correction factor $\Delta$ 


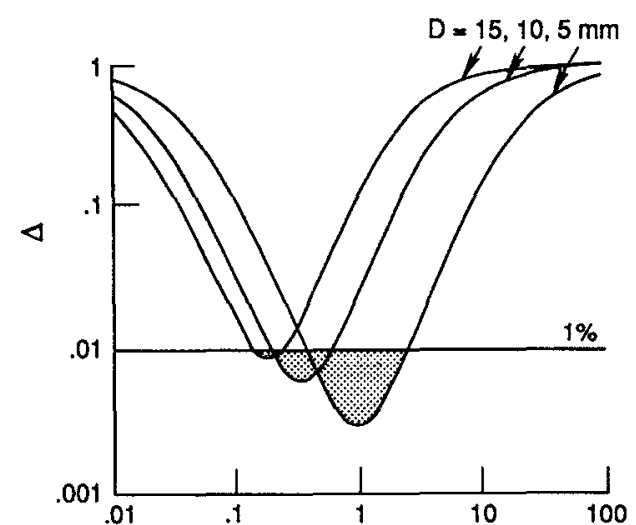

(a)

$\omega(\mathrm{Hz})$

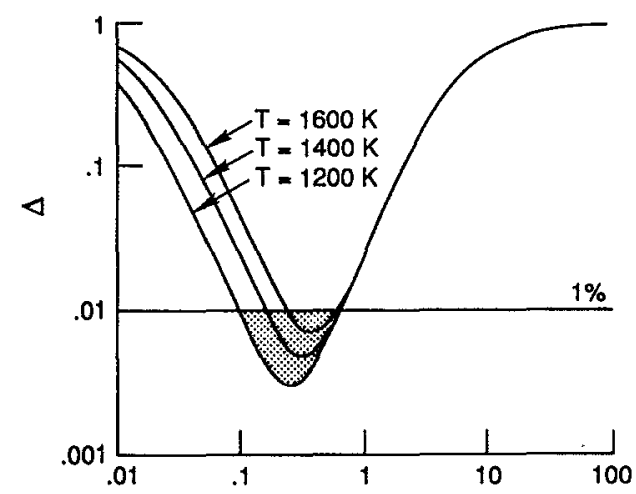

(b)

$\omega(\mathrm{Hz})$
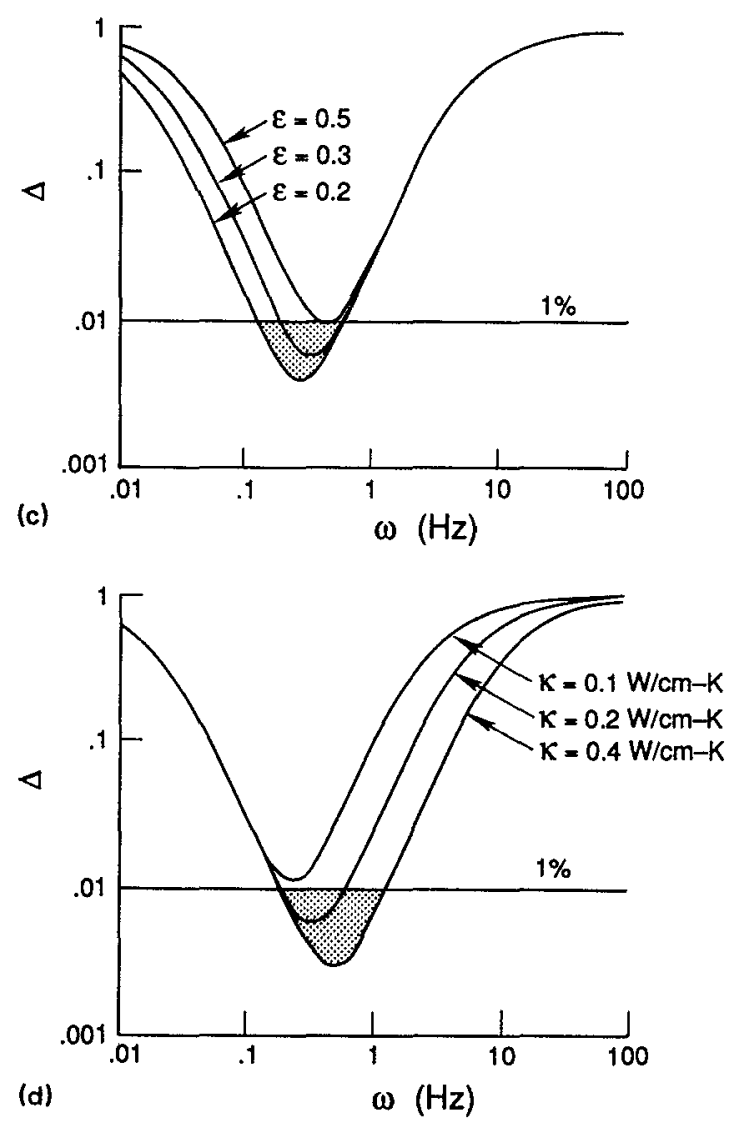

FIG. 5. The correction factor $\Delta$ as function of $\omega$ under the following conditions: (a) various diameters $D$ at $T_{o}=1500 \mathrm{~K}, \epsilon=0.3$ and $\kappa=0.2 \mathrm{~W} / \mathrm{cm}-\mathrm{K}$, (b) various temperatures $T_{o}$ at $D=10 \mathrm{~mm}, \epsilon=0.3$, and $\kappa=0.2 \mathrm{~W} / \mathrm{cm}-\mathrm{K}$, (c) various emissivities $\epsilon$ at $T_{o}=1500 \mathrm{~K}, D=10 \mathrm{~mm}$, and $\kappa=0.2 \mathrm{~W} / \mathrm{cm}-\mathrm{K}$, and (d) various thermal conductivities $\kappa$ at $D=10 \mathrm{~mm}, T_{o}=1500 \mathrm{~K}$, and $\epsilon=0.3$. For frequencies in the range of about $0.5 \mathrm{~Hz}$ the correction factor $\Delta$ can be minimized to less than $1 \%$ indicated by the dashed area.

can be minimized to less than $1 \%$. If $P_{\omega 0}$ and $(\Delta T)_{\omega}$ are known, and the frequency is chosen correctly, the measurement of heat capacity can be performed with an accuracy of better than $1 \%$ without consideration of smaller changes (factor 2 to 3 ) in the emissivity and thermal conductivity. Then the overall accuracy of the measurement is determined by the accuracy of $(\Delta T)_{\omega}$ and $P_{\omega 0}$ determination.

Because $\tau_{1}$ is a sole function of $\epsilon$ (and not $\kappa$ ) and $\tau_{2}$ is a function of $\kappa$ (and not $\epsilon$ ), the frequency can be chosen such as to minimize the influence of one of these parameters on the temperature variation as well. As a consequence, the measured temperature variation according to Eq. (23) can be used further to determine the thermal conductivity if the heat capacity is known. For example, Fig. 5(d) shows the correction factor $\Delta$ as function of frequency of $\kappa$ varying between 0.1 and $0.4 \mathrm{~W} / \mathrm{K}-\mathrm{cm}$ for $\epsilon=0.3, D=10 \mathrm{~mm}$, and $T_{o}=1500 \mathrm{~K}$. For frequencies between 10 and $100 \mathrm{~Hz}$, the $\omega \tau_{2}$-term becomes significant in the $(\Delta T)_{\omega^{*}}$ By correct setting of the input $P_{\omega 0}$, this allows to deduce the thermal conductivity $\kappa$ from the $(\Delta T)_{\omega}$ measurement for the same temperature excursion as for the heat capacity measurement.

\section{ACKNOWLEDGMENTS}

Useful discussions with Dr. D. Herlach (DLR), Dr. R. Willnecker (DLR), and Dr. M. C. Lee (NASA), and the financial support by NASA (NAS 496954MG3203550) are gratefully acknowledged.

${ }^{1}$ E. W. Collings and C. C. Koch, Eds., Undercooled Alloy Phases (The Metall. Soc., Warrendale, PA, 1987).

${ }^{2}$ H. S. Chen and D. Turnball, J. Appl. Phys. 38, 3646 (1967).

${ }^{3}$ H. J. Fecht, J. H. Perepezko, M. C. Lee, and W. L. Johnson, J. Appl. Phys. 68, 4494 (1990).

${ }^{4}$ P. Sullivan and G. Seidel, Phys. Rev. 173, 679 (1968).

${ }^{5}$ R. Bachmann, F. J. Disalvo, T. H. Geballe, R. L. Greene, R. E. Howard, C. N. King, H. C. Kirsch, K. N. Lee, R. E. Schwall, H. U. Thomas, and R. B. Zubeck, Rev. Sci. Instrum. 43, 205 (1972).

${ }^{6}$ H. S. Carslaw and J. C. Jaeger, Conduction of Heat in Solids (Oxford University Press, London, 1959).

${ }^{7}$ E. R. Monazam, D. J. Maloney, and L. O. Lawson, Rev. Sci. Instrum. 60, $3460(1989)$.

${ }^{8}$ E. A. Brandes, Ed., Smithells Metals Reference Book (Butterworths, London, 1983).

${ }^{9} \mathrm{~J}$. Murgrave, Workshop on Containerless Experimentation in Microgravity, Pasadena, Jan. 17-19 (1990). 